\title{
Single-mode waveguide propagation and reshaping of sub-ps terahertz pulses in sapphire fibers
}

\author{
S. P. Jamison, R. W. McGowan, ${ }^{\text {a) }}$ and D. Grischkowsky ${ }^{\text {b) }}$ \\ School of Electrical and Computer Engineering and Center for Laser and Photonics Research, \\ Oklahoma State University, Stillwater, Oklahoma 74078
}

(Received 29 November 1999; accepted for publication 14 February 2000)

\begin{abstract}
Waveguide propagation of sub-ps terahertz pulses in single-crystal sapphire fibers is reported. An incident $\mathrm{THz}$ pulse of approximately $0.6 \mathrm{ps}$ duration undergoes considerable reshaping due to the absorptive and dispersive waveguide propagation, resulting in transmitted chirped pulse durations of 10-30 ps. Good agreement between theory and experiment is obtained by analyzing the propagation in terms of the single $\mathrm{HE}_{11}$ waveguide mode. The dominance of the single $\mathrm{HE}_{11}$ mode, despite the fiber dimensions allowing for multimode propagation, is attributed to the free-space to waveguide coupling. (C) 2000 American Institute of Physics. [S0003-6951(00)02215-4]
\end{abstract}

The technical capability to optoelectronically transmit and detect single cycle pulses of freely propagating $\mathrm{THz}$ electromagnetic radiation has generated much interest in the guided wave propagation of such pulses. At the present time, the highest guided-wave performance has been obtained with a $240-\mu \mathrm{m}$-diam stainless steel waveguide over the frequency range from 0.8 to $3.5 \mathrm{THz}$ and with a power absorption coefficient of less than $1 \mathrm{~cm}^{-1}$. This performance far exceeds that of coplanar and microstrip transmission lines. ${ }^{1}$

An alternative approach would be to use dielectric waveguides. Such waveguides would not have the sharp lowfrequency cutoff of metal waveguides and would thereby extend the low frequency limit of the waveguide. Given a suitable low loss dielectric, such as high-resistivity silicon with a power absorption coefficient of less than $0.05 \mathrm{~cm}^{-1}$ over our frequency range, ${ }^{2}$ dielectric waveguides could have much less absorption than metal waveguides. In addition, due to boundary considerations it should be possible to more cleanly couple, and with a much higher coupling efficiency, linearly polarized $\mathrm{THz}$ radiation into the single dominant mode for dielectric waveguides than for metal circular waveguides. Such dielectric single-mode $\mathrm{THz}$ waveguides would have the promise of an extremely low-loss, flexible interconnect and communications channel, with advantages similar to that of single-mode optical fiber.

Here, we report demonstrations of single-mode propagation of sub-ps $\mathrm{THz}$ pulses in dielectric waveguides (fibers). These demonstrations prove the efficient quasioptical input and output coupling to and from such fibers and show the viability of the single-mode $\mathrm{THz}$ fiber interconnect. The fact that the diameters $(325,250$, and $150 \mu \mathrm{m})$ of the $\mathrm{THz}$ fibers are similar to those of optical fibers, including the core and cladding, gives the $\mathrm{THz}$ fibers similar flexibility and handling properties. Because this work was performed with single-crystal unclad fibers, the waveguide propagation characteristics vary significantly over the extensive frequency spectrum of the nearly single-cycle input pulses, giving rise to considerable absorptive and dispersive reshaping.

\footnotetext{
${ }^{a)}$ Now at: Imation, Oakdale, MN 55128.

b)Electronic mail: danielg@thzsun.ecen.okstate.edu
}

The waveguides used are unclad single-crystal sapphire fibers, with the $c$ axis directed along the length of the fiber, supplied by Saphikon Inc. In our analysis we assume a circular fiber cross-section, although direct examination reveals a slight hexagonal cross-section due to the crystal growth process. All fibers were cut to a length of approximately 8 $\mathrm{mm}$ and polished with diamond film to an optical quality finish on the entrance and exit surfaces.

The experimental arrangement employed for coupling of free-space single-cycle pulses of $\mathrm{THz}$ radiation into the fiber is similar to that used for investigations of $\mathrm{THz}$ propagation in metallic waveguides. ${ }^{1}$ Briefly, the $\mathrm{THz}$ radiation is focused by a silicon lens to a waist diameter of $\approx 200 \mu \mathrm{m}$, with the fiber entrance face placed at the waist position. An identical arrangement is used at the exit face of the sapphire fiber. Reference wave forms and spectra are obtained by removing the sapphire fiber and moving the two silicon lenses to a confocal position. In order to minimize losses due to the mounting arrangement interfering with the fields external to the unclad fibers, the fibers were suspended by the two ends, where each end rested in a hole pierced in a $100-\mu \mathrm{m}$-thick sheet of Mylar oriented transverse to the fiber.

The experimentally obtained wave forms transmitted through the three sapphire fibers are presented in Fig. 1. For all fibers the incident $0.6 \mathrm{ps} \mathrm{THz}$ pulse (shown in the insets) undergoes considerable absorptive and dispersive reshaping and broadening due to the waveguide propagation, and emerges strongly frequency chirped. The degree of chirping is dependent on the fiber diameter, with the $325 \mu \mathrm{m}$ fiber stretching the pulse to $\approx 10 \mathrm{ps}$, while the smallest fiber, 150 $\mu \mathrm{m}$, leads to a pulse duration of greater than $30 \mathrm{ps}$. The differences in initial arrival times for the transmitted pulses in Figs. 1(a)-1(c) are due to variations in the fiber lengths. The decreased signal obtained for the smaller fibers is attributed to the efficiency of coupling into the fibers, which offsets the reduced absorption that is expected at the lower frequencies for the smaller fibers. The amplitude spectrum obtained for transmission through the $325 \mu \mathrm{m}$ fiber is shown in Fig. 2, together with the corresponding reference spectrum. The considerable attenuation of the higher frequencies apparent in Fig. 2 is due to the absorption coefficient of sapphire. 

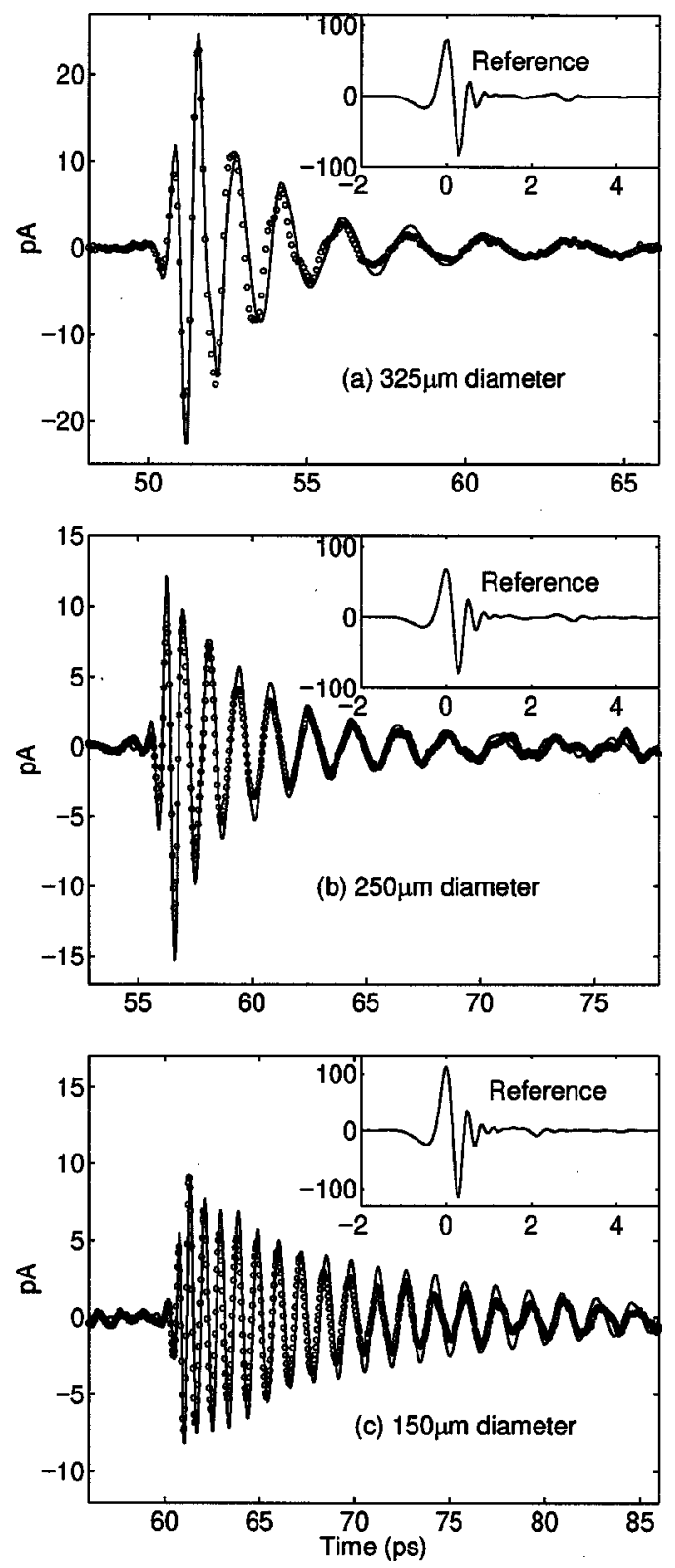

FIG. 1. The THz pulse after passing through (a) 325- $\mu$ m-diam sapphire fiber of length $7.3 \mathrm{~mm}$, and (b) fiber of diameter $250 \mu \mathrm{m}$ and length $7.8 \mathrm{~mm}$, (c) diameter $150 \mu \mathrm{m}$, length $8.3 \mathrm{~mm}$. The solid line is the calculated signal, while the circles $(\bigcirc)$ are the measured pulse. The measured reference signal is shown in the insets.

Calculations have been performed in which the reference pulse (insets of Fig. 1) is subjected to a theoretical propagation through the relevant fiber, resulting in the calculated wave forms that are also shown in Figs. 1(a)-1(c). The calculated transmission spectrum for the $325 \mu \mathrm{m}$ fiber is also shown in Fig. 2. For all three fibers the pulse propagation has been obtained by analysis in terms of the single $\mathrm{HE}_{11}$ waveguide mode. In calculating the waveguide propagation, the reference wave form has been transformed into the frequency domain, where the propagation, attenuation, and coupling are more simply evaluated. Transforming back into the time domain leads to the calculated waveforms of Fig. 1. The dominance of the $\mathrm{HE}_{11}$ mode allows us to express the output electric field for the single mode, in the frequency domain, as

$E_{\text {out }}(\omega)=E_{\text {ref }}(\omega) T C_{11}^{2} \exp \left\{i\left(k_{11}-k_{0}\right) z\right\} \exp \left\{-\alpha_{11} z / 2\right\}$.

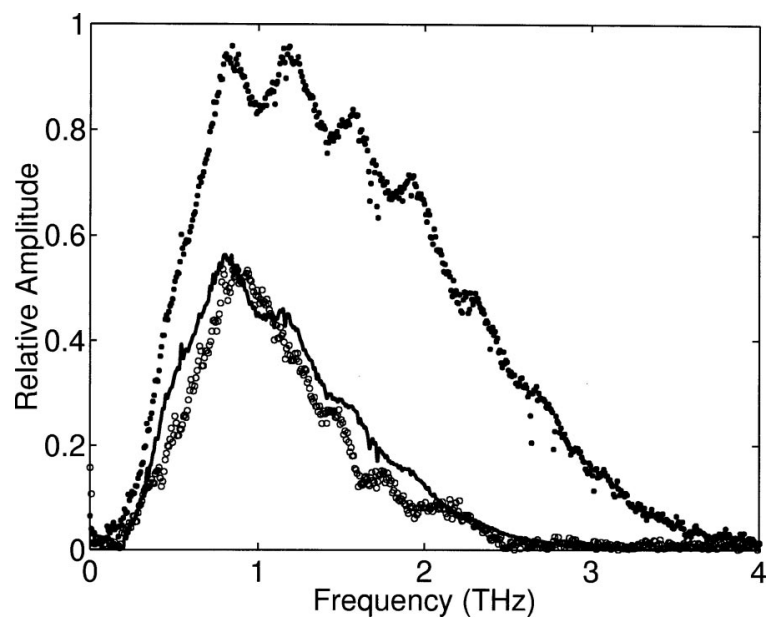

FIG. 2. Amplitude spectra for pulse propagation through a $325-\mu \mathrm{m}$-diam sapphire fiber of length $7.3 \mathrm{~mm}$. The solid line is the calculated spectrum, while the empty $(\bigcirc)$ and filled circles $(\bullet$, upper curve) are the measured transmission and reference spectra, respectively.

The propagation constant of the fibers $\left(k_{11}\right)$ and free-space $\left(k_{0}\right)$ are combined to give the propagation when the freespace path length $z$ is replaced by waveguide propagation over an equal distance. The power absorption coefficient for the $\mathrm{HE}_{11}$ mode is given by $\alpha_{11}$. The transmission coefficient $T$ accounts for losses by reflection at the fiber input and output, while $C_{11}$ is an overlap integral giving the decomposition into the $\mathrm{HE}_{11}$ mode. The second factor of $C_{11}$ accounts for the coupling out of the fiber and onto the detector, for our symmetric and confocal experimental arrangement. The reference spectrum $E_{\text {ref }}(\omega)$ is the Fourier transform of the measured reference pulse $E_{\text {ref }}(t)$.

As the refractive indices of the unclad fiber and the surroundings differ considerably ( $n_{\text {sapphire }} \approx 3.1$ for the ordinary ray $^{2}$ ), the "weakly guiding" approximations commonly employed for optical fibers ${ }^{3}$ are not valid for our experimental situation. To calculate the dispersion of the fiber, the propagation constant was numerically evaluated from the transcendental characteristic equation applicable to the $\mathrm{HE}_{11}$ mode of a dielectric rod with circular cross section. ${ }^{4}$ As the $c$ axis is longitudinal to the fiber, we have taken the refractive index be that of the ordinary ray, neglecting the birefringence of the sapphire. The small frequency dependent variation of the sapphire refractive index was included in the propagation constant calculations, thereby accounting for the material dispersion of sapphire. It is found that the pulse dispersion is almost entirely due to the waveguide propagation characteristics, with the material dispersion playing only a minor role. The group and phase velocities for the 325 and $150 \mu \mathrm{m}$ fibers are shown in Fig. 3, from which it can be clearly observed that the phase velocity approaches that of bulk sapphire at the higher frequencies $(>2 \mathrm{THz})$, while for the very low frequency end of the spectrum the velocity approaches that of free space. This change over is due to the spatial power flow of the fiber changing from containment within the sapphire at high frequencies, to a surface guided wave traveling in the free-space "cladding" of the fiber at low frequencies. For the $150 \mu \mathrm{m}$ fiber the strongly dispersive region can be seen from Fig. 3 to occur in the region of $0.5-2.0 \mathrm{THz}$, which also coincides with the low absorption Downloaded 08 Jan 2008 to 132.163.130.234. Redistribution subject to AIP license or copyright; see http://apl.aip.org/apl/copyright.jsp 


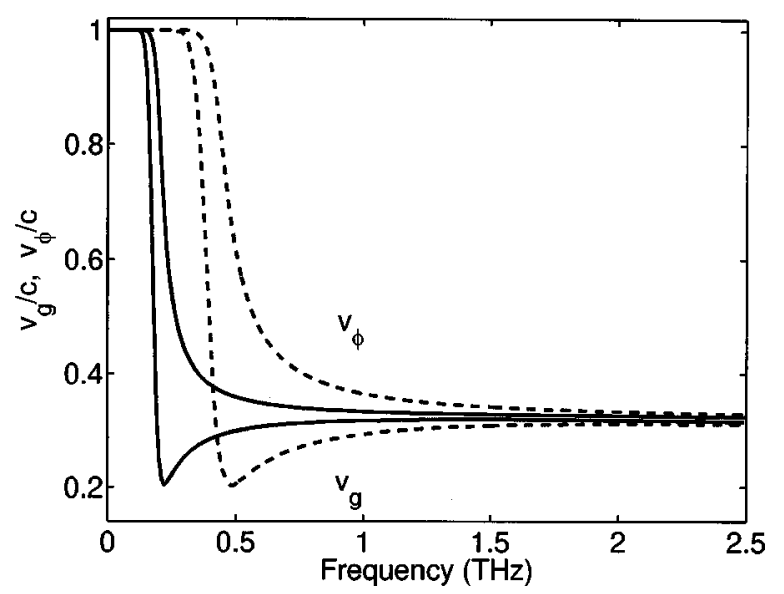

FIG. 3. The calculated group and phase velocities in sapphire fibers of diameter 325 (solid line) and $150 \mu \mathrm{m}$ (dashed line), for the $\mathrm{HE}_{11}$ mode. The upper curves are the phase velocities $v_{\phi}$, while the group velocities $v_{g}$ are given by the lower curves.

region for sapphire and the peak signal detection of our terahertz system. It is to be noted that, in contrast to the waveguide, for bulk sapphire the phase and group velocity are to a good approximation the same, where both decrease with increasing frequency by less than $1 \%$ from low frequencies up to $2.5 \mathrm{THz}$.

The absorption of the guided $\mathrm{THz}$ radiation is in general expected to be dependent on the waveguide propagation. The absorption coefficient $\alpha_{11}$ for the $\mathrm{HE}_{11}$ mode, in terms of the material absorption, has been considered in detail by Elsasser, ${ }^{4}$ whose results have been incorporated into the $\alpha_{11}$ calculated for the pulse propagation, together with the known absorption coefficient for sapphire in the far-infrared. ${ }^{2}$ In the far-infrared the absorption is approximately quadratic with frequency, and leads to an almost total loss of frequencies greater than approximately $2.5 \mathrm{THz}$, as can be seen in Fig. 2 . However, this comparatively large loss is still significantly less than for coplanar and microstrip transmission lines. ${ }^{1}$ For high-resistivity silicon fibers the power absorption coefficient would be less that $0.05 \mathrm{~cm}^{-1} .^{2}$ As shown in Fig. 4,

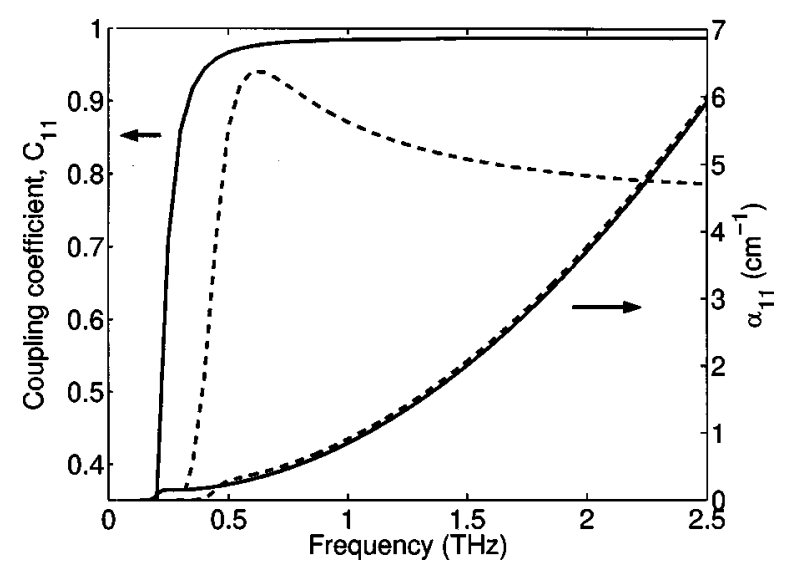

FIG. 4. The power absorption coefficient (right axis, lower curves) and coupling coefficient (left axis) in sapphire fibers of diameter 325 (solid line) and $150 \mu \mathrm{m}$ (dashed line), for the $\mathrm{HE}_{11}$ mode. above $0.5 \mathrm{THz}$ the absorption of the guided wave closely follows that of the ordinary ray in bulk sapphire. However, below 0.2 and $0.4 \mathrm{THz}$ the attenuation is significantly reduced for the 325 and $150 \mu \mathrm{m}$ fibers, respectively. For these lower frequencies a significant fraction of the power of the $\mathrm{HE}_{11}$ mode propagates outside the absorbing fiber, with an associated drop in the propagation losses.

The almost complete dominance of the $\mathrm{HE}_{11}$ mode, despite the fiber dimensions allowing for multimode propagation, is attributable to the efficient, quasioptical, free-space to waveguide coupling. ${ }^{5}$ The coupling coefficient $C_{11}$ has been evaluated through a decomposition of the incident electric field into waveguide modes, where at the fiber input it is given by

$$
C_{m n}(\omega)=\int_{S_{1}} \mathbf{E}_{\text {input }} \cdot \mathbf{E}(\omega)_{m n} d S .
$$

The input field $\mathbf{E}_{\text {input }}$ and the field of the fiber mode $\mathbf{E}_{m n}$ have been normalized. The fields associated with the $\mathrm{HE}_{11}$ mode can be readily calculated once the propagation constant has been determined, ${ }^{3}$ from which the overlap coefficient $C_{11}$ is evaluated. The input beam profile $\mathbf{E}_{\text {input }}$ is assumed to be a frequency independent Gaussian beam with a diameter of $200 \mu \mathrm{m}$. In contrast to metal walled waveguides, the mode patterns $\mathbf{E}_{m n}$ are frequency dependent. The calculated $C_{11}$ are shown in Fig. 4 for both the 325 and $150 \mu \mathrm{m}$ fibers. To include the reflective losses at the entrance and exit planes of the fiber we have approximated the transmission coefficient at each dielectric surface to that of a plane wave. This leads to a total transmission coefficient $T \approx 0.74$ over our frequency range.

In the calculations for the $325-\mu$ m-diam fiber we have not performed any "fitting" of parameters to obtain the best agreement with experiment, with all parameters in the calculation being derived from known, or physically reasonable, parameters. However, for the smaller diameter fibers it was found that the dispersion was consistent only with a slightly larger than nominal diameter. The calculation of Fig. 1(b) has employed a diameter of $266 \mu \mathrm{m}$ (cf. nominal $250 \mu \mathrm{m}$ ), while in Fig. 1(c) the calculation has used a radius of 180 $\mu \mathrm{m}$ (cf. nominal $150 \mu \mathrm{m}$ ). The required increase in radius is assumed to be due to the birefringence of the sapphire, and is interpreted as an effective radius. As can be seen in our comparisons in both the time and frequency domains, we find excellent agreement between theory and experiment.

This work was partially supported by the National Science Foundation and the Army Research Office.

\footnotetext{
${ }^{1}$ R. W. McGowan, G. Gallot, and D. Grischkowsky, Opt. Lett. 24, 1431 (1999).

${ }^{2}$ D. Grischkowsky, S. Keiding, M. van Exter, and Ch. Fattinger, J. Opt. Soc. Am. B 7, 2006 (1990).

${ }^{3}$ J. A. Buck, Fundamentals of Optical Fibers (Wiley, New York, 1995).

${ }^{4}$ W. M. Elsasser, J. Appl. Phys. 20, 1193 (1949). [We note what appears to be a typographical error in the characteristic equation given by D. B. Keck, in Fundamentals of Optical Fiber Communications, second ed. edited by M. K. Barnoski (Academic, New York, 1981), and that this error seems to have been repeated in some optical fiber textbooks.]

${ }^{5}$ A. W. Snyder, IEEE Trans. Microwave Theory Tech. 117, 1138 (1969).
} 